\title{
Computer simulation of industrial hazards ${ }^{1}$
}

\author{
E. G. KNOX \\ Birmingham Health Services Research Centre, Department of Social Medicine, \\ University of Birmingham
}

Knox, E. G. (1973). Brit. J. industr. Med., 30, 54-63. Computer simulation of industrial hazards. A computer simulation system for a range of industrial hazards provided for model experiments which manipulated $(a)$ the sub-structure of an exposed population in terms of agedistributions and levels of exposure, $(b)$ the nature of the dose/response relationship, $(c)$ the latent interval and its variability, $(d)$ normal life-table expectations, and (e) employment turnover rates. The development of the system led to clarification of terms and concepts with ambiguous current usages, notably in relation to latency. Distinction is made between the notions of 'biological' and 'observable' latent intervals.

Hypothetical exercises with the model tested its technical validity and at the same time demonstrated in quantitative terms the relationships between 'biological' and 'observable' latent intervals, employment turnover rates, total mortalities, and the distribution of illnesses and death between those currently employed in the exposing industry, those employed elsewhere, and those retired. Prospects of success for personnel engineering techniques, which manipulate age-distributions of exposed work people in relation to diseases with long latent intervals, were examined.

Published asbestos cancer data were used as a basis for specific model fitting and resulted in a numerical formulation of the exposure/response relationships. Severe exposure results in an increment of risk of death of about $\mathbf{0 . 0 2}$ unit per person per annum for those exposed for around six years, but with higher rates for shorter exposures and lower rates for longer ones. The mean biological latent interval was about 25 years with a coefficient of variation of about $25 \%$. These suppositions explained a range of published data comprehensively and at the same time predicted that $(a)$ persons exposed at severe levels for a working lifetime of 50 years have a $40 \%$ risk of dying from asbestos cancer, and $(b)$ industrial populations with moderate to high turnover rates effect a form of extended dose sharing, and non-linearity of the exposure/response relationship results in substantially more deaths than would be the case if the turnover rate were lower.

Many large-scale industries expose their work people to occupational hazards. Where the effect of the hazard is direct, immediate, and measurable, the conquences of change can be predicted. But where the industry or its processes are new the consequences of exposure may not have been exactly measured, and non-linear relationships, varying durations and

${ }^{1}$ Copies of the program, and specifications, will be provided on request. intensities of exposure, substantial staff turnover rates, and long latent intervals may prevent a quantitative statement of the risk and obstruct the predictions that could be based upon it.

Policy and planning decisions are always based upon predictions. Predictions are mandatory, however difficult and uncertain they may be, and it is important to understand the basis upon which they are made. In formal terms a prediction is always based upon a model, although many models used in 
planning are never exactly stated, existing vaguely in the minds of the planners. Other models are more explicit and must be better than unstated ones if only for purposes of communication. If predictions based upon an explicit model prove wrong then the model can at least be revised; explicit models are the basis of literacy in the world of decision taking. Models are necessary also for extrapolations other than predicting the future, for example to project findings from one industrial situation to another and to provide the general framework of which these situations are examples.

Explicit models are stated in a variety of forms, sometimes as mathematical relationships between variables, sometimes as graphical or diagrammatic relationships, and sometimes as step-by-step specifications of the process whereby one event leads to another. This last form, if expressed in a computer language, can be converted on the computer to a working device, a so-called 'simulation' process, and this is the technique to be followed in this paper.

The provision of a working tool is not the only advantage of this approach; the correct logical statement of the model is assisted by the disciplined and unambiguous syntax of computer language, and in a limited sense the logic can be challenged by offering it to the computer for compilation. The outcome of a modelling process depends jointly upon the premises provided to the program in the form of data and upon the postulated relationships between the premises and consequences as expressed in the program. This separation aids clarity and the working model exercises over data the same discipline which explicit declaration and compilation exercise over the logic of the process; guesses about premises are evidently guesses, and where data are missing they are shown to be missing in a very uncompromising way.

Despite the great theoretical advantages of communicability, generality, discipline, and laboursaving, the pragmatic utility of computer modelling is quite unproven in the particular field considered here. Indeed it has scarcely been tested at all, and this study is possibly a first attempt to use it. The first objective of the study was to construct a simulator capable of describing a range of industrial hazards and to define the necessary inputs. The second was to explore the general properties of the model so produced. The third was to apply the model to a specific case. These objectives are reflected in order of presentation.

\section{Construction of the model}

The model was written in FORTRAN and the data which it requires are presented on punched cards. The main technical alternatives in its construction were between deterministic and stochastic processes and between a dynamic and a steady-state simulation. It was necessary also to devize a method of defining heterogeneous industrial populations and methods of specifying relationships between exposures and consequences.

A deterministic model was preferred for this first approach. With this technique, probabilities are treated as algebraic quantities and manipulated by simple addition, subtraction, and multiplication; hazards and consequences are expressed as fractions and proportions, and the same statements of premises always give the same result. The stochastic approach, generating discrete events on the basis of probabilities and producing different results on different runs, seemed unnecessarily complex for the large-scale predictive purposes envisaged. It is possible, of course, that requirements for a discrete event process could arise in practice, as they have, for example, in the simulation of measles epidemics where a stochastic process was found to give results which matched observations much better than those of deterministic models (Bailey, 1957; Bartlett, 1957). In the longer term the choice of process will depend upon predictive capabilities.

The 'steady state' model was preferred to the 'dynamic', also on grounds of simplicity. The simulated industry is conceived as having reached stability with respect to total size, employment and retirement characteristics, age distribution, subpopulation structure, exposure rates, and doserelated consequences. On this basis a cohort of employees is followed from first recruitment through a working lifetime and into retirement. Change of circumstance with age in a single cohort is readily simulated, but second order changes of the whole process between different cohorts would have introduced a forbidding complexity. However, if an approach to so complex a simulation were required, a series of cohorts of different sizes and under different conditions could be processed separately and the results summated. It may be noted, however, that the advantages of simplicity extend beyond programming and operating; the steady-state simulation proposed affords a degree of standardization and provides a basis for transferring results comprehensibly from one industry or one process or one policy to another.

The method adopted for constructing a heterogeneous industrial population was the separate specification of an arbitrary number (up to 20 , initially) of homogeneous sub-populations. Each must be defined in terms of its relative size, its age distribution, its manpower turnover rate, and its level of exposure to hazard. Age-specific recruitment rates are computed automatically to maintain the steadystate age distribution in the face of staff turnover, death or loss from the hazard in question, and death from other causes. The retirement age must be 
declared and life table data entered, and both are assumed constant over all sub-groups; requirements beyond these assumptions (e.g., for separate treatment of female workers) would require separate runs of the model.

The choice of method for specifying exposure/ response relationships raised deeper issues than those encountered above. There is considerable confusion and ambiguity in current usages of concepts of exposure and response, and a lack of discrimination between distinct implicit models appropriate in different circumstances. At least there is nothing in the terminology of this subject to suggest their clear separation.

One usage supposes that each increment of exposure is related (after an interval) to an increment of risk of disease. However, the hazard itself does not accumulate and, if it is removed, the risk of disease in individuals will (sooner or later) disappear. Examples are industrial accidents, anthrax, and Weil's disease.

Another usage supposes that the hazard accumulates, that an exposed cohort accumulates a progressive risk of subsequent injury which remains after the exposure is removed. Examples are the accumulation of dust in the lungs or radium in the bones.

A general model accommodating both types of relationship, and providing the facility for declaring a latent interval, required the construction of a sequential process passing from exposure, through the intermediate stage of latent injury, to the final stage of ascertained injury. The initial steps necessary to simulation include $(a)$ the declaration of an Annual Exposure Rate (AER) for each sub-population, in units per person per annum; (b) a statement of rules whereby this can be used to derive an Annual Latent Injury Accumulation Rate (ALIAR); (c) a statement of the rules whereby ALIAR interacts with Duration of Exposure in Years (DEY) to compute the current total of Accumulated Latent Injuries (ALI); $(d)$ a statement of the mean Latent Period, its Coefficient of Variation per cent, the percentage of latent injuries which in fact become manifest, and the terms in which the Latent Interval may be modified by age or by the current level of ALI. Stages $(b)$ and $(c)$ above were each governed by the input of five real variables, A, B, C, D, E for the first and F, $\mathrm{G}, \mathrm{H}, \mathrm{J}, \mathrm{K}$ for the second, in the following terms:

$$
\text { ALIAR }=A+B \times A^{-1 E R}+D \times A^{C}{ }^{E}
$$

$\mathrm{ALI}=\mathrm{ALIAR} \times\left(\mathrm{F}+\mathrm{G} \times \mathrm{DEY}^{\mathbf{H}}+\mathrm{J} \times \mathrm{DEY}^{\mathbf{K}}\right)$

Both sets of variables are punched into data cards which may be different on different runs. This permits flexible specification of a wide range of linear and non-linear model relationships, and where a step of the sequence is redundant it may be short-circuited by entering a simple 1:1 relationship. For example, the substitution ALIAR $=0.0+1.0 \times$ AER $^{1.0}+$
$0.0 \times \mathrm{AER}^{1.0}$ could be used in a situation (e.g., in relation to industrial injuries) where the notion of latency is itself redundant.

The declaration of latent intervals was governed by the input of three real variables, $P, Q, R$, also punched into data cards and processed thus:

$$
\text { Latent Interval }=\mathbf{P}+\mathbf{Q} \times \mathrm{AGE}+\mathrm{R} \times \mathrm{ALI}
$$

It was discovered in passing that the apparently simple notion of a latent interval has also been treated ambiguously in the literature of industrial injuries and that several distinct meanings can be attached to the term. A simple biological relationship in time, between an increment of exposure and a subsequent increment of the risk of manifest injury, as used in the model, is not universally understood. Some workers use 'latent injury' to indicate the period between first exposure to hazard and the occurrence of illness in individuals. Others use the interval between first exposure in a group and the first occurrence of a statistically significant excess of disease. Mean observed intervals are necessarily computed on the basis of a specific duration of follow-up, often a truncated period, and even where prolonged followup has been possible it is unusual to see a distinction between 'model intervals' (i.e., 'biological intervals') and the shortened values which result from interactions with natural mortality. Clearly, it is possible to have a constant 'model' mean interval but observed means which vary according to the circumstances in which they are measured.

There is indeed one approach to the effect of exposure to hazard which seems to avoid altogether the notions of discrete injury and of latent interval. It has been supposed by some that radiation exposure will raise the annual risk of cancer in a 30-year-old man to, say, that of a 50-year-old man and that it may continue to rise thereafter as if he were in fact 50 . Here the notion of a latent interval seems to lose its meaning. The simulation system described here will not easily accommodate this kind of relationship.

The program operates as a nested serial summation of small elements. The outermost nest is a summation across the sub-populations and within each subpopulation the age groups are scanned, beginning with the youngest and passing towards the oldest. In order to accommodate possible dependence of latent interval upon age and upon accumulated latent injuries to date (as could be postulated in relation to the consequences of exposure to radiation-see equation 3 above for the mechanism of declaration), each age-set of new recruits to the industrial sub-population is followed sequentially year by year, and latent injury increments are computed repeatedly. For each increment of latent injury there is yet another nest, allocating the consequences of each increment according to the dis- 
tribution of latent intervals. Corrections are applied at this level from a normal life table to delete effects forestalled by death from other causes.

The output, on completion, consists of an age distribution of observed injuries, given separately for workers in the industry, workers now employed elsewhere, and for retired persons. These results, with totals and sub-totals, are given as the annual toll per thousand total workers currently employed, in an industry which has achieved a steady-state situation.

\section{Working properties of the model}

Test runs on hypothetical data

A first series of studies explored the general effects of varying the different input parameters. In part they aimed to validate the model by demonstrating its ability to produce results which were either obvious or computable by simple mathematical methods; and in part they consisted of a search for general conclusions about the importance of different premises. They were not related to any particular industry or occupational hazard. For these purposes the annual exposure rate was defined tautologicallyfor example, as $\mathbf{0 . 0 0 1}$ of a lethal dose-in the sense that this dose, operating linearly and applied for one year to a thousand men, will eventually kill one of them (unless he dies of something else first). In more realistic situations it would, of course, be desirable to express exposure in terms of a directly measured physical unit. Studies of model heterogeneous populations were first carried out to confirm that the program added them together correctly, and the remaining studies were based upon homogeneous populations (i.e., single subsets) in which parameters were varied on different runs.

This range of studies encompassed an examination of the effects of $(a)$ dose variation, $(b)$ different dose/response relationships, $(c)$ different mean latent intervals, $(d)$ different ranges of latent intervals, (e) different industrial turnover rates, $(f)$ different age distributions of the employed populations. Each parameter in turn was provided with a range of values while the others were held constant. The basic set of test parameters consisted of an age distribution with approximately equal numbers in each group, a dose of 0.001 lethal unit per person per annum, a 1:1 linear relationship between AER and ALIAR, a linear dependence of ALI upon ALIAR and duration of exposure, and a mean latent interval of five years with a coefficient of variation of $25 \%$.

With these basic parameters it was not surprising that the result per thousand men employed amounted to very nearly one death per year . . . . in fact $0.956 / 1000 /$ year. Repeat running with a shorter latent interval, one year, resulted in an annual death rate of 0.991 . It is reasonable to suppose that the minor deficiencies are due to deaths from natural causes although there may in addition be some small accumulated rounding-off errors in the summation processes of the model. This is a characteristic artefact of computer integration techniques.

Variation of the dose (AER) between 0.001 and 0.004 by steps of 0.001 gave an exactly linear response and confirmed the proper working of the model. Tests of non-linear relationships also gave results matching those obtained by simple algebra and provided further evidence of technical validity. Linear and non-linear runs gave results which were similar except for the total yield, and in both cases $54 \%$ of the deaths occurred in persons still employed in the industry, $8 \%$ occurred in age-retired persons (including those age-retired from the industry in question and those age-retired from subsequent employment), and $38 \%$ occurred in persons of working age who no longer worked in the industry (see Table 1 for results of linear response to AER = 0.001 ).

The consequences of a power relationship in an industrial situation are considerable. If the exposure rate varies between different sub-populations, the lethal consequences would greatly exceed those for an even distribution of the same total exposure. This contrasts with a linear dose/response relationship, where for a given total population dose exposure variation makes no difference. These facts apply also in reverse, and extrapolations from highly exposed to less exposed groups can result in greatly exaggerated predictions of death and disease if the relationship is assumed to be linear when it is in fact a power relationship. The classic example of this problem is in radiation exposure where the uncertainties of the nature of the relationships are of such overriding importance that no sensible predictions can be made

TABLE 1

Simulated Distribution of Deaths by Age and EMPLOYMENT

(Rate per thousand employed per annum)

\begin{tabular}{l|c|c|c|c}
\hline & Age & Employed & Left & Total \\
\hline & $15-24$ & 0.0311 & 0.0202 & 0.0513 \\
& $25-34$ & $0 \cdot 1133$ & 0.0805 & $0 \cdot 1938$ \\
& $35-44$ & $0 \cdot 1266$ & 0.0915 & $0 \cdot 2181$ \\
& $45-54$ & $0 \cdot 1242$ & 0.0897 & 0.2139 \\
& $55-64$ & 0.1178 & 0.0848 & $0 \cdot 2026$ \\
\hline Sub total & $15-64$ & 0.5130 & 0.3667 & 0.8797 \\
\hline & $65-74$ & 0.0000 & 0.0762 & 0.0762 \\
& Over 75 & 0.0000 & 0.0000 & 0.0000 \\
\hline Grand total & & 0.5130 & 0.4429 & 0.9559 \\
\hline
\end{tabular}

The 'flat-topped' age distribution used for this and similar tests consisted of $4 \%$ in the group $15-19,8 \%$ in the group $20-24$, and $11 \%$ in each of the remaining eight 5-year groups. 
at the present time in relation to large populations suffering low exposures.

The latent interval Long latent intervals reduce specific mortality through the intervention of natural mortality, increase the proportion of events occurring after retirement, and increase the proportion in persons who have left the industry but are still of working age. They interact with industrial age distributions and labour turnover rates so that the effects of varying the latent interval will be different in different industrial circumstances. A first examination was carried out using the basic model values already proposed, that is a flat-topped age distribution, an annual exposure rate of 0.001 , and a linear dose/response relationship. Latent intervals were varied between zero and 40 years by five-year intervals, using a constant coefficient of variation of $25 \%$; results are set out in Table 2.

Lengthening the latent interval up to about 10 years had only a small effect upon total deaths and it was not until the interval had increased beyond 20 years that successive increments resulted in substantial falls in mortality. However, the effect upon deaths in current employment (assuming a labour turnover rate of $10 \%$ per annum) was immediate and rapid. With a latent interval of five years only $54 \%$ of all cases occurred in employees; a 10-year interval reduced this to $29 \%$, and a 20 -year interval to less than $10 \%$. Deaths among age-retired persons did not overtake those in current employees until the latent interval passed 14 years, and the most dramatic ratio shift for early increments of latent interval (say, up to 8 years) was between deaths in those still employed in the industry and those employed elsewhere. This is a function of the labour turnover rate as well as the latent interval and we shall return to this point later. An important practical conclusion

\section{TABLE 2}

Simulated Death Rates in Employed and Retired Persons according to Different Mean LATENT INTERVALS

\begin{tabular}{c|c|c|c|c}
\hline $\begin{array}{c}\text { Mean latent } \\
\text { interval }\end{array}$ & $\begin{array}{c}\text { Total deaths } \\
\text { per thousand } \\
\text { per annum }\end{array}$ & $\begin{array}{c}\text { Deaths in } \\
\text { specific } \\
\text { employ- } \\
\text { ment }\end{array}$ & $\begin{array}{c}\text { Deaths } \\
\text { among } \\
\text { retired }\end{array}$ & $\begin{array}{c}\text { Deaths among } \\
\text { those working } \\
\text { elsewhere }\end{array}$ \\
\hline 0 & 1.000 & 1.00 & 0.00 & 0.00 \\
5 & 0.956 & 0.51 & 0.07 & 0.37 \\
10 & 0.893 & 0.26 & 0.14 & 0.49 \\
15 & 0.807 & 0.13 & 0.18 & 0.49 \\
20 & 0.709 & 0.07 & 0.20 & 0.44 \\
25 & 0.597 & 0.03 & 0.20 & 0.36 \\
30 & 0.490 & 0.02 & 0.20 & 0.27 \\
35 & 0.386 & 0.01 & 0.19 & 0.18 \\
40 & 0.295 & 0.01 & 0.17 & 0.12 \\
\hline
\end{tabular}

for the present is the extent of the calibration which is shown to be necessary for actual morbidity estimates based upon employed persons only, whenever the latent interval exceeds a year or two.

These examinations also provided some information about the quantitative relationships between biological (i.e., model) latent intervals and mean intervals as measured in practice. In practical situations, ascertained cases are likely to be biased towards shorter intervals and estimates of means will tend to be low; this can in fact be demonstrated from the model. The mean age for all the elements of exposure in the tests discussed above was 41.6 years. The mean age at death for a model latent interval of 35 years was 65.6 years, so that with complete follow-up the observable mean interval was only 24 years. The bias introduced by natural mortality is, therefore, 11 years, almost one-third of the model value. If observed cases had been limited to those still in employment the observed mean age at death would have been only 57.7 years and the observed mean interval only 15.1 years: this is less than half of the 'model' value. Other things being equal, the later the age at exposure the greater will be the discrepancy between the forward-looking model interval and a retrospectively measured mean interval, and we must expect that data collected in different ways will produce widely different results even when they are investigating the same biological process.

Experiments in varying the coefficient of variation of the latent interval showed only small effects. There was a drop in total mortality as the range was widened from 5 towards $80 \%$ (for a mean of 15 years) but the maximum reduction achieved was just a little over $10 \%$. Deaths among age-retired persons remained almost constant. For those of working age, the ratio of deaths still in the industry to deaths elsewhere rose as the coefficient was increased; this was probably due to a compressing effect of the computational method at the lower end of the gaussian distribution, giving a decreased opportunity for workers to change jobs between the exposure and the subsequent event.

Labour turnover rate The main effect expected from an increased labour turnover rate is a decrease in the ratio between those affected while still in their current employment and those who have moved to jobs elsewhere. Provided the relationship between exposure and result is linear, the turnover rate should have no effect upon total subsequent deaths, and provided that the accumulated dose has no effect upon latent interval, the ratio between deaths in those employed and deaths in retired persons should not be affected.

On the other hand, a non-linear relationship would interact with the turnover rate, a high rate producing a form of extended dose-sharing, and the total mor- 
tality would change in response. In the case of a power function, such as that discussed earlier, an increased turnover would have a beneficial effect; but in a situation where the early increments of exposure were the most dangerous (as in exposure to industrial accident risks), an increased labour turnover rate would increase the number of deaths. For a first examination of labour turnover rates, a linear dose/response relationship was used with a mean latent interval of five years and a $25 \%$ coefficient of variation. A range of labour turnover rates was examined from $5 \%$ per annum to $30 \%$ per annum, by $5 \%$ intervals, and results are given in Table 3 . There was a small fall of total mortality as the turnover rate increased but this is attributed to a discontinuity effect in the model which left short gaps in the population exposure as employees left and were replaced. The extent of this change was less than $1 \%$. The ratio of deaths in the industry to deaths in employment elsewhere decreased very rapidly as the employment turnover rate was increased.

Provided that the distribution of latent intervals is symmetrical, we would expect the ratio of deaths occurring inside and outside the parent industry to reflect the total numbers of initial employees still inside and now outside the industry at about the time the deaths occurred. This can be computed by converting the labour turnover rate to a 'proportion retained' after the passage of one latent interval. In Table 4 different annual labour turnover rates are translated into such proportions and are compared with the inside/outside death ratios obtained by runs of the model; the correspondence is reasonably accurate. There may be instances where this simple relationship between annual turnover rate, biological latent interval, and percentage of deaths (or onsets) occurring while still employed in the industry should provide a means of computing any one of these variables when the value of the others is known.

TABLE 3

Simulated Death Rates in Employed and Retired Persons, according to Different ANnual Staff TURNOVER RATES

\begin{tabular}{c|c|c|c|c}
\hline $\begin{array}{c}\text { Annual turn- } \\
\text { over rate } \\
\%\end{array}$ & $\begin{array}{c}\text { Total deaths } \\
\text { per thousand } \\
\text { per annum }\end{array}$ & $\begin{array}{c}\text { Deaths in } \\
\text { specific } \\
\text { employ- } \\
\text { ment }\end{array}$ & $\begin{array}{c}\text { Deaths } \\
\text { among } \\
\text { retired }\end{array}$ & $\begin{array}{c}\text { Deaths among } \\
\text { those working } \\
\text { elsewhere }\end{array}$ \\
\hline 5 & 0.957 & 0.68 & 0.08 & 0.20 \\
10 & 0.956 & 0.51 & 0.08 & 0.37 \\
15 & 0.955 & 0.38 & 0.08 & 0.49 \\
20 & 0.954 & 0.28 & 0.08 & 0.59 \\
25 & 0.953 & 0.21 & 0.07 & 0.67 \\
30 & 0.952 & 0.15 & 0.07 & 0.73 \\
\hline
\end{tabular}

TABLE 4

Comparison between Proportion of Deaths OCCURRING IN CURRENT EMPLOYEES, AND AN Estimate based UPON PROPORTION OF EMPloyees STILL IN EMPLOYMENT ONE LATENT INTERVAL AFTER RECRUITMENT

\begin{tabular}{c|c|c}
\hline $\begin{array}{c}\text { Annual } \\
\text { turnover } \\
\text { rate \% }\end{array}$ & $\begin{array}{c}\text { Deaths in employment } \\
\text { as percentage of all } \\
\text { induced deaths during } \\
\text { working age, i.e., } \\
\text { before 65th birthday }\end{array}$ & $\begin{array}{c}\text { Percentage of } \\
\text { employees still in } \\
\text { employment after one } \\
\text { latent interval of } \\
\text { years }\end{array}$ \\
\hline 5 & 76.7 & $77 \cdot 4$ \\
10 & $58 \cdot 3$ & $59 \cdot 0$ \\
15 & 43.8 & $44 \cdot 4$ \\
20 & 32.6 & $32 \cdot 8$ \\
25 & 23.9 & $23 \cdot 7$ \\
30 & $17 \cdot 3$ & 16.8 \\
\hline
\end{tabular}

${ }^{1}$ For a latent period of $n$ years and a turnover rate of $\mathrm{x} \%$, the percentage of employees still in the industry is computed as

$$
100\left(1-\frac{x}{100}\right)^{n}
$$

Age distribution of the population Older workers exposed to industrial hazards with long latent intervals will die from causes other than the specific consequences of the hazard more often than will younger workers. Consequently, industries offering such hazards might take steps to employ older rather than younger people in dangerous occupations. The effect of such a personnel engineering policy was investigated by comparing two extreme population distributions, one with a very high preponderance of younger workers and the other with a high preponderance of older ones. In the first the percentage distribution in successive five-year age groups from 15 years was, $30,25,15,10,5,5,5,3,1,1$, and in the second it was $1,1,3,5,5,5,10,15,25,30$. It is difficult to imagine any real population with either distribution but results from such extremes provide insight into the limits to which policies of this type could be effective. The dose/response relationship was assumed linear, the annual exposure rate set at 0.001 unit, and the labour turnover rate at $10 \%$ per annum. A range of mean latent intervals was explored and the results are set out in Table 5.

These results show that for short latent intervals, any practical manipulation of the age distribution would not have a substantial effect upon total mortality; for a latent interval of five years the total specific mortality in the older population was $92 \%$ of that in the younger population. For longer intervals the effect was greater; with a 15 years' latent interval the mortality in the older population was only $68 \%$ of that of the younger population, and for 
TABLE 5

Effect of Population Age Structure on Total Deaths AND THeIR Distribution

\begin{tabular}{l|c|c|c|c|c}
\hline $\begin{array}{l}\text { Popula- } \\
\text { tion } \\
\text { type }\end{array}$ & $\begin{array}{c}\text { Latent } \\
\text { interval } \\
(y r)\end{array}$ & $\begin{array}{c}\text { Total } \\
\text { deaths } \\
\text { per } \\
\text { thousand } \\
\text { per } \\
\text { annum }\end{array}$ & $\begin{array}{c}\text { Deaths } \\
\text { in } \\
\text { specific } \\
\text { employ- } \\
\text { ment }\end{array}$ & $\begin{array}{c}\text { Deaths } \\
\text { among } \\
\text { retired }\end{array}$ & $\begin{array}{c}\text { Deaths } \\
\text { among } \\
\text { those } \\
\text { working } \\
\text { else- } \\
\text { where }\end{array}$ \\
\hline Young & 5 & 0.988 & 0.57 & 0.01 & 0.41 \\
Old & 5 & 0.911 & 0.41 & 0.21 & 0.29 \\
Young & 15 & 0.945 & 0.19 & 0.03 & 0.72 \\
Old & 15 & 0.646 & 0.07 & 0.36 & 0.22 \\
Young & 25 & 0.853 & 0.06 & 0.08 & 0.71 \\
Old & 25 & 0.362 & 0.01 & 0.23 & 0.12 \\
Young & 30 & 0.782 & 0.04 & 0.11 & 0.63 \\
Old & 30 & 0.260 & $<0.01$ & 0.17 & 0.08 \\
\hline
\end{tabular}

mean latent intervals of 25 and 30 years the proportions reduced to $42 \%$ and $33 \%$. Deaths at different ages might deserve different 'costings' but in purely numeric terms it is clear that personnel engineering techniques of this kind can be reasonably effective only if the latent interval is very long indeed. For shorter latent intervals the main effects of these manipulations were upon the numbers who were affected while still in employment, either inside or outside the industry. For a five-year mean latent interval, $99 \%$ of all deaths in the younger population occurred during current or subsequent employment, but only $77 \%$ of those in the older population. When the 15 -year interval was used the figures were $96 \%$ and $45 \%$.

The combined effects of a long latent interval, a moderate labour turnover rate, and an older worker policy could virtually banish the consequences of a hazard from the population currently employed in the industry. For the longest latent intervals (see last four lines of Table 5) this will happen in any case without troubling to manipulate the age distribution of the workers employed.

However, at intermediate ranges (e.g., 15 years' mean latent interval) the deaths occurring in employees can be reduced several fold by an older worker policy, even in the absence of a substantial effect upon total deaths. Extrapolation of the results for the 15-year interval to an industry employing 10000 older workers distributed by age as suggested above, and with a $10 \%$ labour turnover, shows that over a five-year period it might ascertain three or four deaths among its current employees from the specific hazard in question but might fail to ascertain about 30 deaths in ex-employees.
Application of the model to a particular case, asbestos and cancer

A search of the literature for factual material applicable to the model revealed how scarce such information is. There are very few occupational hazards where the range of published investigations could be related within a common model framework, translatable freely from one industrial situation to another. Indeed there are $\mathrm{f} \in \mathrm{W}$ instances where a quantitative relationship of any kind can be established between a particular hazard or employment pattern and the health consequences. The published material on asbestos exposure in relation to cancer is one of the reasonably documented exceptions. While displaying gaps, it permits an attempt at integration.

Many studies have shown high incidence and death rates from cancer among those exposed occupationally to asbestos dust. This is in addition to high death and disability rates from asbestos fibrosis of the lungs. The tumours include cancers of the lung and intestinal tract, and thoracic and abdominal mesotheliomas. Cancer of the lung is the most important numerically, and the rate in asbestos workers is several times that for the normal population.

The evidence suggests that responses to differing severities and durations of exposure are not linear. Newhouse (1969) measured increased total cancer rates in workers exposed for less than two years, and they were not much increased following more prolonged exposure. In addition, she detected high mesothelioma rates in those exposed at home only to dust from the clothing of asbestos workers (Newhouse and Thompson, 1965) which suggests that for this tumour small exposures can be effective for some people. In such a situation, dose sharing as effected by a high employment turnover rate would be harmful to the population as a whole.

An exact statement of the numerical relationship between exposure and consequence is not yet possible, but Newhouse's (1969) data provide a basis for first estimates. She followed 689 employees exposed for less than two years to 'severe' dust levels and obtained cancer data for periods of follow-up ranging from 17 to 31 years. There were 17 cases of cancer compared with 4.3 expected for a similar non-exposed group. Thus, about 13 cases resulted from less than $2 \times 689$ person-years' exposure. On a linear basis this could be expressed as 0.01 to 0.02 lethal unit per year of exposure. Workers exposed initially for more than two years revealed 18 cases among 471 so exposed, compared with an expected value of 4.1 cases. The mean exposure period was not stated, but if it was four to six years the annual exposure could be expressed as 0.007 to 0.01 lethal unit per annum. Newhouse failed to observe any statistical excess of deaths from malignant disease 
within 16 years of the first exposure, so deaths related to the later years of prolonged exposures were certainly incomplete. Both numerical estimates, and especially the second, must be regarded as minima.

It is possible to modify these first linear estimates of the numerical relationships in a manner expressing the apparent non-linear facts, for example as follows :

ALIAR $=(0 \cdot 0+0 \cdot 3$ AER $+0 \cdot 1 \sqrt{ }$ AER $)$

$\operatorname{ALI}=\operatorname{ALIAR}(0.0+0.5 \mathrm{DEY}+1 \cdot 0 \sqrt{\mathrm{DEY}})$

These relationships express falling yields with increasing intensities and durations of exposure. The relationship expressed in equation 4 is entirely arbitrary, as exposure intensity was not expressed in physical terms; it is designed to be equivalent to a $1: 1$ linear relationship when AER is set at 0.02 , to enhance the effect at lower 'doses', and to attenuate the effect at higher ones. The duration-of-exposure relationship (equation 5) has a similar general shape but is designed to be equivalent to a 1:1 linear relationship, for exposures of exactly four years. Substitution of AER $=0.02$ in equations 1 and 2 gives higher cancer rates, ultimately, than those observed by Newhouse within available follow-up periods and assumes, very approximately, that about two-thirds of potential injuries resulting from short exposures had so far appeared in Newhouse's material but only about one-third of the injuries resulting from longer exposures.

The justification for such an assumption depends on estimates of biological latent intervals. Newhouse found no evidence of excess cancer before 16 years after the first exposure, and although her longest follow-up period extended to 31 years we are not told how many patients were actually followed for this time. Her observed values must represent an underestimate of the biological mean interval distribution both by virtue of the cases which had not yet occurred and the cases which would never appear because of natural mortality. On the other hand, she used intervals since first exposure as her criterion, which for prolonged exposures would tend to overestimate the true interval. Hammond, Selikoff, and Churg (1965) examined 57 cancers in relation to onset of exposure and found a mode in the range 30-34 years; 20 intervals were greater than 40 years. Hueper (1965) collected cases from the literature and found mean latent periods in five different countries ranging from 23 to 28 years. The range of observations is therefore considerable and the variation evidently related to the mode of ascertainment and the duration of follow-up. A model value of 25 years, although arbitrary, is compatible with these various sets of data, and an attributed coefficient of $25 \%$ would bring the lower tail of the distribution down to about 13 years (i.e., the third percentile approximately).

Simple substitution of AER $=0.02$ in formula 4 (above) predicts that $3.0 \%$ of men exposed for one year would eventually get an asbestos cancer if they did not first die of something else (compare $1.9 \%$ in Newhouse's follow-up period for men exposed for less than two years) and $10.8 \%$ of those exposed for six years (compare $3.0 \%$ ). A man employed from age 15 to 65 would have a 50-year exposure which, applied directly to the formula, gives him a $64 \%$ chance of either developing asbestos cancer or having a latent asbestos cancer by the time he dies.

The interaction of the 25-year latent interval with natural mortality was tested in the last case by running the model on a population distributed with $10 \%$ in each of 10 five-year age groups and a zero labour turnover rate. In a steady state this population would have an annual intake of 20 recruits per annum at age 15. The result was an annual mortality of $9 \cdot 24$ deaths per 1000 workers currently employed, that is 9.24 deaths per 20 recruits. This suggests that $46 \%$ would actually die of their cancer and the balance, to $64 \%$, would die of something else first. In fact . the value of $46 \%$ is itself an exaggeration because the model replaces workers who have left or who have died, and even when the labour turnover rate is set to zero, as in the above run, the high mortality (threequarters before retirement) results in substantial replacements. The implication of model fitting to the Newhouse data is therefore an eventual observable asbestos cancer mortality of around $40 \%$ in those most severely exposed for periods of 50 years.

By any standards $40 \%$ is a very high figure indeed, similar to the results of exposure to 2-naphthylamine. One of the purposes of simulation techniques is to bring data-based premises and consequences into confrontation, and this alarming figure might be taken to throw doubt upon the observations. However, there are other data to suggest that the framework of the prediction is substantially correct.

Elmes (1971) followed up a group of Belfast insulation workers with severe exposure to asbestos dust in 1940. Details of previous and subsequent exposure were not given, although cross-sectional sampling tends to identify those with relatively prolonged exposures. After 26 years they found that 98 were dead compared with a natural mortality expectation of 37 , and that 45 were dead from cancer compared with an expectation of $6 \cdot 8$. Therefore, 38 may be said to have died from 'asbestos cancer', an interim minimum of $22.5 \%$ of the group. If the ratio of 38 asbestos cancers to 37 total expected mortality were to continue, the predictions of the model would be borne out. The only apparent perturbation is through asbestos deaths not due to cancer.

Attempts to extend studies from model relationships applicable in scientific studies to realistic simulations of industrial environments raised further data problems. Little relevant information is available about the age distributions of employed persons, 
or their employment turnover rates, and few workers have found it possible to express severities of exposure in physical units translatable from one set of circumstances to another, or to say how the populations they studied were distributed in terms of severity of exposure.

Enterline and Kendrick (1967) gave the age distribution of a population studied in the United States. It was a younger population than that of the total employed population in the United Kingdom (Central Statistical Office, 1970), a difference partly attributable to different national age profiles, but also no doubt to the rapid growth in recent years of the asbestos industry. For purposes of a simulation study an intermediate distribution was chosen, constructed in fact from two equal parts, the first with Enterline and Kendrick's distribution and the second with that for the United Kingdom total.

Declaration of the employment turnover rate was based upon a frank guess; no recorded data were found which could be related to age and severity of exposure in this industry. The value chosen was a turnover rate of $10 \%$ per annum overall and this was partitioned to a rate of $15 \%$ per annum for the younger age profile group and $5 \%$ per annum for the older group. Because of the non-linear dose/ response relationship adopted, the effect of choosing too low a value will be to underestimate subsequent death rates; too high a choice will overestimate. It was necessary also to be arbitrary in constructing a distribution of levels of exposure. An annual exposure rate of 0.02 was attributed to a severely exposed group and a lower value, one-third of this, was attached to a 'moderately' exposed group. Slight exposures were ignored altogether so that results must be regarded as referring to injuries per thousand industrial population working in moderate to severe grades of exposure (in Newhouse's terms) and not per thousand total industrial population. For purposes of the test the severely and moderately exposed groups were supposed to be equal in size; both the younger high turnover group and the older low turnover group were divided into equal parts for this purpose, resolving the total test population into four sub-groups. It would follow that if, in a particular circumstance, the attributed ratio of severe to moderate is grossly inaccurate, the outcome will have to be modified.

One other point was taken into consideration. Either age or accumulated previous exposure might affect the latent interval; in the case of industrial bladder cancer, for example, it has been noted that workers with late first exposure tend to have short latent intervals. One explanation there was that such workers were given relatively responsible jobs with high exposures but other explanations seem more likely. It might be an artefact of limited opportunity for follow-up or due to a differential truncation of longer potential intervals by natural mortality. Alternatively, it could arise from a genuine rise in susceptibility or a change in the rate of development of the pathological process with age. The latter explanations of the finding might be transferable to the asbestos hazard. In sympathy with these considerations, but perhaps superfluously, the asbestos/ cancer model was modified by reducing the mean latent interval of 25 years by one-tenth of the age at exposure. Thus, for a man exposed at 20 years of age a mean interval of 23 years is attributed to that increment of exposure, and at 50 years of age a mean interval of 20 years.

The results of the computation based upon the above premises are given in Table 6 . The overall result was 10.37 deaths per annum in employees and ex-employees per thousand current employees. This is greater than the earlier result, for a zero turnover population, all exposed at the 'severe' level for 50 years. It appears that with the dose/response relationships envisaged, a moderate turnover rate of $10 \%$ has an effect so serious that it more than compensates for a two-thirds reduction in exposure in one-half of the population. Only $14.1 \%$ of the deaths occurred in current employees, and even at preretirement ages this figure was only $16 \cdot 2 \%$.

The modal value for age at death was 50 years and the mean age 53.1 years, and these values afford some independent validation of the simulation. They correspond well with Buchanan's (1965) estimate of 55.2 years for men with lung cancer following asbestos exposure. For women his mean age was $44 \cdot 6$, which might reflect a younger age distribution at

TABLE 6

Simulated Distribution of Asbestos Cancer DEATHS

\begin{tabular}{c|c|c|c|c}
\hline $\begin{array}{c}\text { Age } \\
(y r)\end{array}$ & $\begin{array}{c}\text { In current } \\
\text { employment }\end{array}$ & $\begin{array}{c}\text { In other } \\
\text { employment } \\
\text { or retired }\end{array}$ & Total & $\begin{array}{c}\text { Percentage } \\
\text { in current } \\
\text { employment }\end{array}$ \\
\hline-25 & 0.01 & 0.01 & 0.01 & 45.8 \\
-30 & 0.02 & 0.03 & 0.06 & 34.6 \\
-35 & 0.08 & 0.21 & 0.29 & 26.3 \\
-40 & $0 \cdot 17$ & 0.68 & 0.85 & $19 \cdot 8$ \\
-45 & 0.24 & 1.32 & 1.56 & 15.6 \\
-50 & 0.26 & 1.71 & 1.98 & 13.3 \\
-55 & 0.25 & 1.64 & 1.90 & 13.2 \\
-60 & 0.23 & 1.21 & 1.44 & 16.0 \\
-65 & 0.21 & 0.76 & 0.97 & 21.7 \\
\hline Sub total & 1.47 & 7.57 & 9.04 & 16.2 \\
\hline-70 & 0 & 0.64 & 0.64 & 0 \\
-75 & 0 & 0.43 & 0.43 & 0 \\
-80 & 0 & 0.21 & 0.21 & 0 \\
Over 80 & 0 & 0.06 & 0.06 & 0 \\
\hline Total & 1.47 & 8.90 & 10.37 & $14 \cdot 1$ \\
\hline
\end{tabular}


exposure, although he does not say so. Hueper (1965) assembled mean ages from reports published from several countries. The values were 50 years in the United States of America, 57 years in Canada, 54 years in England, and 53 years in Germany.

\section{Discussion and conclusions}

The simulation approach is in some ways the inverse of standard analytic practice. Conclusions are derived by iteration of premises and comparison of consequences with observations, rather than computed directly from the observations themselves. This is difficult to present sequentially within the standard format of an analytic scientific report and is reflected in some untidiness of conclusion. The main function of a reasonably validated simulation system is to predict, and where the predictions are long-term the usefulness of the approach is difficult to justify in terms of immediate result. However, the main conclusions of the present application are as follows:

(1) Many apparently various sequences of exposure to industrial hazard, and the subsequent events, can in fact be accommodated within a general framework of not too forbidding complexity. The relationships of the sequence, and the premises which need to be declared, can be defined exactly.

(2) Ambiguities in the common usages of certain terms and concepts were revealed by this process of definition. In particular, the meaning of latent interval' has been interpreted in several different ways. The idea of a 'model' or 'biological' latent interval is distinguished in the present context as a general concept, which of its nature is not capable of direct computation. All values based on observations and described as latent intervals are to be regarded as special and inherently biased estimates.

(3) Schematic inputs to the general model demonstrated the relative effects of variation in each of the main factors. Quantitative estimates were made of discrepancies between 'model' and 'measured' latent intervals, and a simple approximate mathematical relationship was suggested between latent interval, employment turnover rate, and the proportion of injuries occurring during current employment. The limits and circumstances of possible success with personnel engineering policies were also demonstrated.

(4) Literature searches revealed few industrial situations documented to the extent necessary for exact simulation. Insofar as prediction was shown to depend upon information not commonly available, we can conclude that few current industrial hygiene policies can be both comprehensive and soundly based.

(5) The hazards of asbestos dust exposure are documented better than many industrial hazards, and while simulation of the asbestos cancer problem still encountered data deficiencies, they did not prevent the construction of a reasonable simulation study. The main conclusions here were:

(a) The biological latent interval from exposure to death from asbestos cancer is about 25 years. Results from a range of studies with different computed mean intervals can be explained by supposing an approximately symmetrical distribution of the latent interval with a coefficient of variation of $25 \%$.

(b) Exposure at the most severe levels recently reported is equivalent to an accumulating risk of 0.02 lethal unit per annum with respect to asbestos cancer. This value applies to exposures lasting around four years, but the risk in relation to duration is higher for shorter exposures and less for longer ones.

(c) Exposure for a working lifetime at severe levels results in a $40 \%$ risk of death from asbestos cancer. A probable non-linear relationship between intensity and duration of exposure on the one hand and consequences on the other implies that the extended dose-sharing effected by labour turnover is harmful to the population as a whole. A mobile industrial population exposed to mean dust levels much less than the worst could readily accumulate more deaths in the long run than a population universally exposed at the worst levels, but with a zero labour turnover rate.

\section{References}

Bailey, N. T. J. (1957). The Mathematical Theory of Epidemics. Griffin, London.

Bartlett, M. S. (1957). Measles periodicity and community size. J. roy. statist. Soc., Series A. 120, 48-60.

Buchanan, W. D.'(1965). Asbestos and primary intrathoracic neoplasms. Ann. N.Y. Acad. Sci., 132, 507-518.

Central Statistical Office (1970). Annual Abstract of Statistics. No. 107, Table 133, p. 122. H.M.S.O., London.

Elmes, P. C. (1971). International classification of radiographs of pneumonoconioses Brit. J. industr. Med., 28, 93-96.

Enterline, P. E., and Kendrick, M. A. (1967). Asbestos-dust exposures at various levels and mortality. Arch. environm. Hith, 15, 181-186.

Hueper, W. C. (1965). Occupational and nonoccupational exposures to asbestos. Ann. N.Y. Acad. Sci., 132, 184-195.

Hammond, E. C., Selikoff, I. J., and Churg, J. (1965). Neoplasia among insulation workers in the United States with special reference to intra-abdominal neoplasia. Ann. N.Y. Acad. Sci., 132, 510-525.

Newhouse, M. L. (1969). A study of the mortality of workers in an asbestos factory. Brit. J. industr. Med., 26, 294-301. , and Thompson, H. (1965). Mesothelioma of pleura and peritoneum following exposure to asbestos in the London area. Brit. J. industr. Med., 22, 261-269.

Received for publication March 14, 1972. 